\title{
La justification dans l'enseignement de la lecture : étude exploratoire auprès d'enseignant[e]s de français du 1er cycle du secondaire québécois
}

\author{
Marie-Hélène Forget \\ Université du Québec à Trois-Rivières
}

Marie-Christine Beaudry

Université du Québec à Montréal

\section{Résumé}

La justification est requise dans le travail en lecture en classe de français au secondaire québécois, notamment, pour justifier son interprétation, sa réaction ou son appréciation d'un texte. Or, on en connait peu sur les usages qu'en font les enseignant[e]s dans le cadre de leur enseignement de la lecture. Une recherche exploratoire menée auprès de 12 enseignant[e]s de français a mis au jour des besoins de clarifier ce que constitue la justification en classe de français et d'outiller les enseignants pour en faire un usage plus diversifié et mieux arrimé aux besoins d'apprentissages des élèves en lecture.

Mots-clés : justification, lecture, méthodes d'enseignement, activités scolaires 


\begin{abstract}
Justification is required in reading in French class in Quebec' high schools, in particular to justify interpretation, reaction or appreciation of a text. However, little is known about the uses that teachers make of justification in the context of their teaching of reading. Exploratory research conducted with 12 French teachers has revealed needs to clarify what constitutes justification in French class to equip teachers to make more diversified use of it that is better aligned with students' learning needs.

Keywords: justification, reading, teaching method, school activities
\end{abstract}

\title{
Introduction
}

Dans le programme de français langue d'enseignement du 1er cycle du secondaire québécois (MEQ, 2006), on indique que la compétence des élèves en lecture de 
textes courants et littéraires « repose sur la qualité de la compréhension, la justesse de l'interprétation et la justification des réactions » et qu'elle « se conjugue étroitement avec l'appréciation de ces textes » (p. 97). C'est donc le travail sur ces quatre dimensions combinées de la lecture qui doit permettre aux élèves de développer leur compétence en lecture. Ce travail passe par des demandes de justifications qui consistent, pour l'élève, à indiquer comment ou pourquoi il en vient à formuler telle ou telle interprétation d'un texte, pourquoi il réagit de telle ou telle autre manière à la lecture d'un texte ou pourquoi il adopte telle ou telle autre position relativement aux qualités d'un texte. Ces demandes de justifications sont sollicitées lorsque l'enseignant place les élèves en situation de confronter leur interprétation ou de partager leur appréciation d'un texte dans le cadre de discussions, lorsque l'enseignant[e] questionne les élèves (à l'oral ou à l'écrit) à propos de leur interprétation d'un texte ou lorsque les élèves sont appelés à rédiger un commentaire de lecture, par exemple. Dans ces contextes, la justification se présente tantôt comme un outil pour apprendre à lire (lors des discussions), tantôt comme un outil de vérification et d'évaluation des compétences en lecture (dans des tâches de type « questionnaire »), tantôt comme un objet d'enseignement (lors de l'enseignement des genres à dominante justificative, comme l'appréciation littéraire).

Bien que la justification soit associée de près au travail en lecture dans les programmes d'enseignement du primaire et du secondaire québécois, peu de références didactiques existent pour soutenir les enseignants - et, par conséquent, les élèves lorsque vient le temps de faire usage de la justification. Qui plus est, aucune recherche n'a porté, à notre connaissance, sur l'usage fait par les enseignant[e]s de la justification dans l'enseignement de la lecture ni sur son rôle dans l'apprentissage de la lecture au secondaire québécois. Pour en avoir une meilleure idée, nous avons mené une première recherche exploratoire auprès de 12 enseignant $[\mathrm{e}] \mathrm{s}$ de français du $1 \mathrm{er}$ cycle du secondaire.

Cet article rend compte des résultats de cette recherche exploratoire qui nous informe davantage sur le point de vue d'enseignant[e]s relativement à ce que constitue la conduite de justification, aux capacités de leurs élèves à justifier leurs propos ainsi qu'aux usages déclarés de la justification dans le cadre de leur enseignement de la lecture. L'article se termine sur des constats qui ouvrent vers des perspectives de recherche quant à la conduite de justification dans l'enseignement de la lecture et le développement de la compétence en lecture des élèves québécois. 


\section{La justification dans le travail en lecture au secondaire québécois}

La justification est une conduite discursive qui sert à répondre à la question « Pourquoi affirmes-tu cela ? » (Chartrand, 2013). Dans le programme de français langue d'enseignement du primaire québécois (MEQ, 2001), on indique que l'élève devrait « réagir [aux textes lus ], c'est-à-dire exprimer et justifier les émotions, les sentiments, les opinions qui émergent au cours de la lecture » (p. 74). Pour parvenir à déployer son appréciation, l'élève « apprend aussi à se doter de critères pour poser des jugements critiques et esthétiques sur les œuvres ainsi que pour justifier ses appréciations » (p. 84). Dans la progression des apprentissages au secondaire ${ }^{1}$ (PDA) (MELS, 2011), on précise que : «Au primaire, l'élève fait l'expérience de la justification : il a été appelé à justifier, oralement ou par écrit, [entre autres,] son interprétation d'un texte, en s'appuyant sur des extraits du texte » (p. 17). Ainsi, l'élève qui arrive du primaire a dû développer un savoir d'expérience de la justification, ayant été placé dans des situations de communication et d'apprentissage en lecture requérant des justifications de sa part.

Dans le programme québécois de français langue d'enseignement du 1er cycle du secondaire (MEQ, 2006), la justification est présentée comme un véritable savoir disciplinaire (Chevallard et Johsua, 1985/1991). Associée au déploiement de la pensée critique, et ce autant en lecture et en écriture qu'en communication orale, on y indique que l'élève doit, par exemple, justifier un choix, une interprétation, une réaction, une opinion ou une appréciation, confronter des positions ou établir un consensus, partager des perceptions, des représentations ou émettre des recommandations en les justifiant (MEQ, 2006). Dans la PDA (MELS, 2011), on précise que la justification vise à exprimer le bienfondé d'un propos de manière à le rendre recevable aux yeux de son destinataire, définition que l'on trouve également dans quelques travaux en didactique du français qui ont porté sur cet objet (Chartrand, 2013 ; Garcia-Debanc, 1994, 1996). Toujours dans la PDA (MELS, 2011), on en présente les attributs essentiels : une affirmation ou un jugement est présenté, suivi des raisons qui fondent ce propos ; divers procédés

1 La Progression des apprentissages au secondaire (MELS, 2011) est un document complémentaire au programme officiel destiné à préciser et à programmer l'enseignement et l'apprentissage des savoirs disciplinaires selon une progression cohérente. 
(citer, définir, comparer) et connecteurs (car, parce que, puisque) peuvent être utilisés. On y indique également que la justification se retrouve dans deux « domaines ». Dans le domaine du « savoir », la justification sert à présenter les étapes du raisonnement qui viennent valider la réponse à une question. Pour bien la distinguer de l'explication, on y spécifie que :

« Contrairement à l'explication, [la justification] ne vise pas à faire comprendre en rendant visibles les relations de causalité entre des éléments [...]. La justification ne fait qu'expliciter à postériori la démarche cognitive (le raisonnement) qui a conduit à l'expression du propos » (p. 21).

Dans le domaine de la « subjectivité », elle est associée à des genres tels que la quatrième de couverture ou l'appréciation critique, et sert à fournir les raisons qui fondent la position d'un élève sur un élément du texte ou sur un texte. Pour bien la distinguer de l'argumentation, on y précise que la justification « ne cherche pas à convaincre le destinataire d'adopter une thèse sur un sujet controversé où s'affrontent deux thèses opposées », comme c'est le cas de l'argumentation selon ces précisions (p. 21). La justification vise plutôt à faire valoir la légitimité d'un propos ou d'une réponse à une question. Ainsi, la justification est une conduite essentiellement subjective (Fasel-Lauzon, 2009) dans la mesure où elle donne à voir la perspective personnelle de l'énonciateur sur l'objet de son propos. Dans le travail en lecture, la justification vise donc à faire valoir, aux yeux de son destinataire, le bienfondé de son interprétation, de sa réaction ou de son appréciation des textes lus, en fournissant les raisons qui la fondent (Garcia-Debanc, 1994).

Notre savoir d'expérience d'enseignantes au secondaire, puis de professeures et de superviseures de stage en formation des maitres, nous a permis d'observer que les enseignant $[\mathrm{e}] \mathrm{s}$ se servent de la justification pour suivre et vérifier l'état des compétences des élèves en lecture, notamment en ce qui concerne leurs interprétations, leurs réactions et leurs appréciations. Certaines questions sollicitant des justifications sont en effet régulièrement posées aux élèves (oralement ou par écrit) pour s'assurer qu'ils ont lu les textes et pour les évaluer (Falardeau, 2003 ; Jorro, 1999). Pourtant, bien que la justification figure dans les programmes, autant au primaire qu'au secondaire, notre savoir d'expérience nous amène à faire le constat que les enseignant[e]s demeurent peu outillé[e]s pour soutenir leurs élèves lorsque vient le temps de les inviter à justifier 
telle réaction, interprétation ou appréciation. Les enseignant $[\mathrm{e}] \mathrm{s}$ recourent bien à divers dispositifs didactiques — comme le cercle de lecture (Hébert et al., 2015 ; Hébert et Lafontaine, 2012) ou le journal de lecture (Ahr et Joole, 2013) — pour amener leurs élèves à développer leurs compétences en lecture, ces dispositifs impliquant l'usage de la justification de la part des élèves, mais sans que cette conduite ait fait l'objet d'une attention particulière dans le cadre des recherches sur le sujet. Les enseignant[e] $\mathrm{s}$ montrent également à leurs élèves à produire des textes à dominante justificative (Forget, 2012, 2017) à la suite de la lecture d'une œuvre (production d'un commentaire littéraire) ou d'un corpus de textes (production d'un commentaire sur un évènement, un lieu, etc.). Encore ici, les recherches consultées n'ont pas précisément mis la focale sur les liens entre le développement des compétences en lecture et l'écriture de justifications. À notre connaissance, aucune recherche ne s'est par ailleurs intéressée à la justification comme moyen d'accéder aux raisonnements des élèves en lecture comme c'est le cas dans certaines recherches portant sur l'enseignement de la grammaire (Fisher et Nadeau, 2014 ; Tallet, 2003). Ainsi, peu de données nous permettent de bien connaitre le statut, la

place et le rôle de la justification dans l'enseignement de la lecture, même si elle se trouve associée de près au travail en lecture, qu'elle fait partie des critères d'évaluation de la compétence en lecture, qu'elle constitue un objet à enseigner au 1er cycle du secondaire au Québec et qu'elle fait l'objet d'une épreuve obligatoire en écriture en deuxième secondaire, épreuve qui repose sur la lecture d'un corpus de textes desquels découlera un commentaire justifié de la part de l'élève.

C'est dans ce contexte que nous nous sommes donné comme objectifs de : 1) cerner la compréhension d'enseignant[e]s de français relativement à l'objet « justification », 2) connaitre leurs points de vue quant aux capacités à justifier de leurs élèves et 3 ) explorer les usages déclarés de cette conduite dans le cadre de leur enseignement de la lecture en classe de français.

\section{Une recherche exploratoire pour sonder les enseignant $[\mathrm{e}] \mathrm{s}$}

Chacun des objectifs de cette recherche exploratoire porte sur l'un ou l'autre des trois pôles du triangle didactique : le pôle « objet », le pôle « apprenants » et le pôle « enseignement » (Dabène, 2005). Ces trois pôles sont constitutifs de la relation 
didactique qui s'établit dans les différents contextes d'enseignement et d'apprentissage, relation dont découlera un certain apprentissage d'un objet chez des apprenants en fonction de l'enseignement octroyé, pour le dire trop simplement. Par ailleurs, on admet, avec Dabène, que les contextes social et éducatif, les savoirs disciplinaires et didactiques accessibles ainsi que les représentations et les pratiques liées aux usages de la langue constituent des facteurs d'influence importants de cette relation didactique, ce à quoi n'échappent évidemment pas les premiers responsables de la relation didactique, c'est-àdire les enseignant[e]s. Comme ces derniers jouent effectivement un rôle de premier chef dans l'établissement et la direction que prendra la relation didactique qui a cours dans leur classe, il est apparu important d'accéder à leur point de vue à propos de ces trois pôles : la justification comme « objet », les capacités à justifier des « apprenants » (leurs élèves) auxquelles ils ajustent leur enseignement, et les usages déclarés de la justification dans le cadre de leur « enseignement» de la lecture.

Pour atteindre ces trois objectifs, nous avons mené une recherche exploratoire auprès de 12 enseignant[e]s de français du 1er cycle du secondaire réparti[e]s dans 5 commissions scolaires de 3 régions du Québec (écoles publiques comprenant des classes dites ordinaires et des classes du $\mathrm{PEI}^{2}$ ) et une école privée. Ces personnes ont été recrutées par le biais de conseillères pédagogiques que nous avons contactées. Le tableau 1 dépeint cet échantillon de convenance.

\section{Tableau 1}

Répartition des participant[e]s selon l'expérience et le milieu scolaire

\begin{tabular}{ccccc}
\hline $\mathrm{Nb}$ d'années d'expérience & 4 ans & 10 à 15 ans & 16 à 20 ans & Plus de 20 ans \\
\hline Classes ordinaires d'écoles publiques & 2 & 2 & 3 & 1
\end{tabular}

2 Cet acronyme désigne le Programme d'éducation international (PEI) de l'Organisation du baccalauréat international (OBI) qui est jumelé, dans ces classes, au Programme de formation de l'école québécoise et constitue ainsi un programme d'enrichissement. 


\begin{tabular}{lcccc}
\hline \multicolumn{1}{c}{ Nb d'années d'expérience } & 4 ans & 10 à 15 ans & 16 à 20 ans & Plus de 20 ans \\
\hline $\begin{array}{l}\text { Classes PEI d'écoles publiques } \\
\text { Classes d'écoles privées }\end{array}$ & 1 & 1 & & 1 \\
\hline Total & 3 & 3 & 1 & \\
\hline
\end{tabular}

Nous avons demandé aux participant[e]s de sélectionner deux activités de lecture qu'ils soumettent à leurs élèves et qui impliquent, de leur point de vue, un usage de la justification (pôle « enseignant»). Nous avons donc recueilli 24 activités, mais avons dû en rejeter 3 parce qu'elles ne constituaient pas une activité de lecture comportant une tâche justificative. Au total, 21 activités ont été retenues pour analyse. Nous avons également sollicité un entretien individuel d'une heure au cours duquel nous leur avons demandé :

- Comment définissent-ils la justification et comment la présentent-ils à leurs élèves (pôle « objet ») ;

- Quel est leur avis sur les forces et les besoins d'apprentissage de leurs élèves relativement à cette conduite (pôle « apprenants »);

- Comment s'en servent-ils en classe pour enseigner la lecture (pôle « enseignant »).

Pour préciser ces usages déclarés, nous leur avons enfin demandé de nous présenter les deux activités choisies, de préciser le contexte de leur réalisation, leur déroulement et leur finalité (pôle « enseignant »). Les entretiens ont eu lieu à l'hiver 2019, dans leur lieu de travail, au moment qui leur convenait.

Les données ont été analysées qualitativement dans un contexte où, d'un côté, peu de données de recherches existent sur le sujet, et de l'autre, la justification est présente dans les programmes québécois et fait même l'objet d'une épreuve nationale obligatoire. De plus, comme il s'agit d'une recherche exploratoire, nous avons opté pour une approche inductive (Karsenti et Savoie-Zajc, 2004) sans grille de codage ou catégories préexistantes, de sorte à nous donner les moyens de saisir les catégories des participant[e] s telles qu'elles se donnent (Paillé et Mucchielli, 2016). Nous présentons dans ce qui suit le détail du traitement et de l'analyse des données.

Une fois les entretiens réalisés, nous en avons fait plusieurs écoutes afin de transcrire, dans un tableau, l'essentiel du propos des participant $[\mathrm{e}] \mathrm{s}$, et ce, pour chaque bloc de questions posées. Les données ont ensuite fait l'objet d'une analyse s'inspirant 
de la méthode d'analyse thématique (Paillé et Mucchielli, 2016), méthode qui convient bien aux recherches de type exploratoire. Cette méthode consiste en la « transposition d'un corpus donné en un certain nombre de thèmes représentatifs du contenu analysé » (p. 162) relativement aux objectifs de recherche. Le chercheur va " procéder systématiquement au repérage, au regroupement et [...] à l'examen discursif des thèmes abordés dans le corpus » (Paillé et Mucchielli, 2016, p. 162). Pour le repérage, nous avons repassé les données à la recherche d'éléments répondant aux objectifs de notre étude, puis procédé à un étiquetage des éléments repérés, étiquetage qui a pris la forme de mots-clés inscrits en marge du tableau, représentatifs du propos du [de la] participant[e]. Nous avons ensuite regroupé les données dont les étiquettes s'avéraient identiques ou semblables, ce qui a enfin permis de formuler les thèmes présents dans le discours des participant[e]s relativement aux objectifs de la recherche. Le tableau 2 présente un exemple de données transcrites (colonne 2), puis étiquetées à l'aide de mots-clés (colonne 3 ) et finalement regroupées selon le thème (colonne 4).

\section{Tableau 2}

\section{Exemple d'analyse de données}

\begin{tabular}{|c|c|c|c|}
\hline Participant[e] & Données transcrites & Mots-clés & Thème \\
\hline $\begin{array}{l}\text { Ens. } 7 \\
(1: 34 \text { à } 9: \\
20)\end{array}$ & $\begin{array}{l}\text { Justifier c'est d'être en mesure de venir appuyer leurs per- } \\
\text { ceptions, leur compréhension et appuyer par des extraits } \\
\text { des œuvres ou par leur vécu. La justification pour moi } \\
\text { c'est un « appui », c'est venir appuyer sa réponse. }\end{array}$ & $\begin{array}{l}\text { Appuyer sa } \\
\text { réponse (extraits } \\
\text { de texte, vécu) }\end{array}$ & Action \\
\hline $\begin{array}{l}\text { Ens. } 3 \\
(5: 30 \text { à } 12 \\
: 40)\end{array}$ & $\begin{array}{l}\text { Pour l'élève, la justification c'est être capable d'expliquer } \\
\text { quelque chose en apportant des preuves, en faisant les } \\
\text { liens avec ses connaissances générales ou théoriques. }\end{array}$ & $\begin{array}{l}\text { Expliquer } \\
\text { en prouvant } \\
\text { (connaissances } \\
\text { générales) } \\
\end{array}$ & Action \\
\hline $\begin{array}{l}\text { Ens. } 10 \\
(5: 00 \text { à } 12 \\
: 22)\end{array}$ & $\begin{array}{l}\text { En lecture, la justification c'est d'être capable de faire un } \\
\text { lien entre soit le texte ou soi-même, aller chercher dans le } \\
\text { texte pourquoi tu dis ça. Il faut montrer le « "comment » } \\
\text { tu en es arrivé à ça. Quand on entre dans l'implicite, dans } \\
\text { l'inférence, alors là il faut aller chercher qu'est-ce qui } \\
\text { s'est passé comme connexion dans ta tête, quels liens tu } \\
\text { fais. }\end{array}$ & $\begin{array}{l}\text { Montrer le rai- } \\
\text { sonnement (liens } \\
\text { effectués) }\end{array}$ & Action \\
\hline $\begin{array}{l}\text { Ens. } 9 \\
(6: 30 \text { à } 16 \\
: 10)\end{array}$ & $\begin{array}{l}\text { J'indique les étapes à suivre en utilisant les critères : } \\
\text { - reformuler l'essentiel de la question ; } \\
\text { - donner un extrait du texte ou un exemple de ta vie ; } \\
\text { - faire une mini-conclusion. }\end{array}$ & $\begin{array}{l}\text { Étapes à suivre } \\
\text { pour répondre à } \\
\text { une question }\end{array}$ & Structure \\
\hline
\end{tabular}




\begin{tabular}{|c|c|c|c|}
\hline Participant[e] & Données transcrites & Mots-clés & Thème \\
\hline $\begin{array}{l}\text { Ens. } 2 \\
(4: 53 \text { à } 20 \\
: 00)\end{array}$ & $\begin{array}{l}\text { Je leur montre qu'il y a quatre étapes : la réponse « selon } \\
\text { moi », ensuite une preuve pertinente tirée du texte, en- } \\
\text { suite l'explication, puis une reformulation à la fin. Donc il } \\
\text { y a au moins quatre phrases. }\end{array}$ & $\begin{array}{l}\text { Étapes à suivre } \\
\text { pour répondre à } \\
\text { une question }\end{array}$ & Structure \\
\hline $\begin{array}{l}\text { Ens. } 1 \\
(1: 00 \text { à } 9 \text { : } \\
20)\end{array}$ & $\begin{array}{l}\text { Je leur montre ce qu'il y a dans la PDA: } \\
\text { - j'ai une opinion que je dois présenter ; } \\
\text { - j'amène des exemples pour « expliquer » pourquoi je } \\
\text { pense cela; } \\
\text { - je réaffirme mon opinion. }\end{array}$ & $\begin{array}{c}\text { Étapes à suivre } \\
\text { lors de l'écriture } \\
\text { d'un texte }\end{array}$ & Structure \\
\hline
\end{tabular}

Pour nous assurer de la validité des analyses effectuées, les résultats de chaque étape ont été confrontés entre les deux chercheuses pour « aboutir ainsi à une certaine représentation du réel par la mise en évidence des écarts ou convergences de représentations » (Krief et Zardet, 2013, p. 215). Ces confrontations, ou « intersubjectivités répliquées » (Husserl, 1992), sont essentielles pour développer une compréhension commune nécessaire au travail d'analyse. En outre, le matériel didactique employé dans le cadre des activités que nous ont présentées les enseignant[e]s a été utilisé comme données de triangulation permettant notamment de corroborer les usages déclarés des enseignant[e]s. Les sections suivantes présentent les résultats de nos analyses.

\section{La justification dans l'enseignement de la lecture en classe de français : le point de vue d'enseignant [e]s}

Dans les sections qui suivent, nous présentons les résultats obtenus relativement aux trois pôles du triangle didactique : les définitions que donnent les participant[e]s de l'objet « justification », les capacités des élèves à justifier du point de vue de ces enseignant[e]s et les usages déclarés de la justification dans leur enseignement de la lecture.

\section{Comment des enseignant[e]s définissent-ils la justification ?}

Les enseignant[e]s ayant participé à cette recherche définissent la justification de deux manières : soit il s'agit d'une action menée par l'énonciateur avec, pour certains enseignant $[\mathrm{e}] \mathrm{s}$, une intention d'agir sur autrui ; soit ils la définissent à partir des attributs textuels qui la caractérisent. Précisons que ces définitions cohabitent chez ces 
enseignant[e]s et sont associées aux différents usages qu'ils [elles] font de la justification auprès de leurs élèves.

Dans le cas de la première définition que nous avons pu thématiser, les enseignant[e]s associent la justification à une « action », qu'ils nomment de cinq façons distinctes. Les trois premières nomment une action qui se place du côté de l'énonciateur décrivant en quelque sorte ce que fait ce dernier lorsqu'il justifie : 1) appuyer ses propos à l'aide de preuves, 2) expliquer ses propos à l'aide de preuves, 3) montrer son raisonnement. Les verbes d'action « appuyer », « expliquer » et « montrer » utilisés par les participant[e]s indiquent la nature de l'action « justifier » de leur point de vue. Les deux autres façons de nommer la justification représentent une action dont l'intention est d'agir sur quelqu'un : 4) parvenir à convaincre quelqu'un d'agir, ou 5) expliquer à quelqu'un l'intérêt d'une chose. Ces définitions impliquent le destinataire de façon plus marquée, celui sur lequel la justification vise à agir. En effet, les termes « parvenir », « convaincre » et « intérêt » évoquent la recherche d'une forme d'adhésion de quelqu'un d'autre à ce qui lui est présenté. Cette première définition est associée par les enseignant[e]s à des contextes de discussions entre élèves, ou de travail sur l'interprétation, la réaction ou l'appréciation. Elle reprend des éléments énoncés dans la PDA (MELS, 2011) et dans le programme de français langue d'enseignement au secondaire (MEQ, 2006) selon lesquels la justification vise entre autres à exprimer le bienfondé de son propos afin de le rendre recevable pour un destinataire.

Une deuxième définition dégagée des discours et du matériel fournis par les enseignants[e]s associe de manière plus directe la justification à une structure textuelle. Lorsqu'il est question de faire réaliser aux élèves des productions écrites justificatives découlant de tâches préalables en lecture (l'appréciation littéraire par exemple) ou d'évaluer leur compétence en lecture par l'entremise de questions d'interprétation et de réaction, c'est la structure textuelle à laquelle l'élève doit se conformer qui est présentée comme représentative de ce qu'est la justification. C'est ici que les enseignant[e]s évoquent des critères de qualité pour reconnaitre une bonne justification : sa structure doit présenter une affirmation ou une réponse, des preuves tirées du texte, des liens avec le vécu de l'élève, des marqueurs de relation utilisés adéquatement, un vocabulaire précis et connoté. Quand les enseignant[e]s évoquent cette définition de la justification, il n'est alors plus question, selon leurs discours, de la situation de communication, du destinataire ou de l'intention de communication. Au contraire, la justification correspond 
essentiellement à ces attributs textuels, linguistiques et discursifs que l'élève doit utiliser à bon escient pour formuler, à l'écrit, ses réponses aux questions d'interprétation, de réaction ou d'appréciation. Enfin, il apparait que cette définition prend rapidement le pas sur l'autre dans les discours des enseignant[e]s et les activités qu'ils soumettent à leurs élèves lorsqu'ils évoquent les moments d'évaluation. Selon nous, la raison en est que cette seconde définition de la justification est beaucoup plus opérationnelle, offrant des éléments observables aux enseignant[e]s qui peuvent ainsi plus facilement évaluer les productions écrites et les réponses des élèves. Par contre, cette définition plus technique semble gommer l'aspect communicationnel de la justification dont la qualité textuelle peut pourtant dépendre.

Il ressort de ces résultats que deux définitions de la justification cohabitent chez les enseignant[e]s : l'une, évoquée en relation avec des contextes d'apprentissage de l'interprétation, de la réaction et de l'appréciation, met l'accent sur l'intention de communication propre à la justification ; 1'autre, évoquée en relation avec des contextes d'évaluation en lecture et en écriture, met l'accent sur ses attributs discursifs, textuels et linguistiques observables. Par ailleurs, l'analyse a mis en lumière un recours indifférencié chez certains participants des termes « expliquer » et « justifier», utilisés alors comme des synonymes. D'autres participant [e]s déclarent que, de leur point de vue, une explication « sert à » justifier une réponse. On peut donc penser que la distinction entre « expliquer » et « justifier » telle que présentée dans le programme d'études (MEQ, 2006) et la PDA (MELS, 2011) reste à clarifier pour ces enseignant[e]s. D'autres déclarent ne pas bien cerner les frontières entre la justification et l'argumentation, montrant peut-être que les précisions offertes à ce sujet dans les prescriptions ministérielles ne sont pas tout à fait éclairantes. Nous aborderons maintenant les perceptions des enseignant[e]s relatives aux capacités de leurs élèves à justifier leurs propos.

\section{Quel est l'avis des enseignant [e]s relativement aux capacités à justifier de leurs élèves ?}

Aux dires de ces enseignant[e]s, leurs élèves montrent de bonnes capacités à justifier, notamment dans le cadre d'interactions orales. Les enseignant[e]s précisent que leurs élèves sont assez habiles pour justifier lorsque le sujet abordé est près de leurs intérêts et de leurs connaissances ; ils ont également une facilité à prendre position. Si ces 
conditions sont réunies dans le cadre d'un travail en lecture, il semble que les élèves soient capables de faire des liens entre le texte et leurs connaissances, et de personnaliser leur réponse. Ils comprennent également rapidement le but de la justification et son utilité. Une recherche de Forget (2014), qui a montré des capacités certaines à justifier chez des élèves du secondaire dans de telles conditions, corrobore ces observations formulées par les enseignant[e]s.

Les enseignant[e]s relèvent par ailleurs que ces capacités liées à la justification s'amenuisent quand le sujet des textes est plus loin de la réalité des élèves et que les repères nécessaires à leur compréhension ne font pas partie de leurs connaissances. Ils [Elles] mentionnent également de plus grandes difficultés à justifier leurs réponses à l'écrit, l'absence de preuves, la difficulté de produire des réponses personnalisées et appuyées, et les liens boiteux. Si les élèves semblent plus habiles à rendre compte de leur interprétation ou de leur réaction à l'oral, c'est probablement dû aux relances des interlocuteurs, à leurs demandes de clarification ou à leurs désaccords qui les amènent à devoir approfondir leur réponse et donc leur raisonnement. L'absence d'interaction en situation d'écriture devient potentiellement un obstacle à cet approfondissement, ce que corrobore la recherche depuis fort longtemps (Dabène, 1991). Néanmoins, certains ont souligné que les élèves apprennent facilement la justification et qu'ils progressent rapidement dans les apprentissages relatifs à la justification écrite lorsqu'elle leur est systématiquement enseignée. D'autres considèrent cet apprentissage comme étant plus difficile, notamment pour les élèves qui manquent de repères et de connaissances pour comprendre les textes lus.

\section{Quels usages des enseignant[e]s font-ils[-elles] de la justification dans le cadre de l'enseignement de la lecture ?}

L'analyse a révélé certains constats généraux quant aux usages déclarés de la justification dans l'enseignement de la lecture. D'abord, la justification est sollicitée sous deux formes : à l'oral, dans des contextes le plus souvent dédiés à l'apprentissage ; puis à l'écrit, dans des contextes liés de plus près à l'évaluation. La justification est également sollicitée le plus souvent au travail en lecture de textes littéraires (romans, nouvelles, poèmes, textes de chanson) selon les données recueillies. En effet, seulement 4 activités sur les 21 retenues proposaient un travail à partir de la lecture de textes courants. Les sections 
suivantes présentent les usages déclarés de la justification par les enseignant $[\mathrm{e}] \mathrm{s}$ lors de tâches d'enseignement-apprentissage de la lecture. Le tableau 3 présente les catégories qui ont émergé de l'analyse.

\section{Tableau 3}

Catégories issues de l'analyse des données

\begin{tabular}{|c|c|c|}
\hline $\begin{array}{l}\text { Répondre à des questions } \\
\text { (sans enseignement de la } \\
\text { justification) }\end{array}$ & $\begin{array}{l}\text { Répondre à des questions } \\
\text { (avec enseignement de la } \\
\text { justification) }\end{array}$ & Rédiger des textes justificatifs \\
\hline \multirow[t]{4}{*}{ Questionnaires (6) } & Questionnaires (3) & \multirow{4}{*}{$\begin{array}{c}\text { Rédaction (2) d'une appréciation } \\
\text { littéraire } \\
\text { Lecture d'une œuvre }\end{array}$} \\
\hline & Enseignement de la justification & \\
\hline & Observation d'exemnles et de & \\
\hline & contrexemples & \\
\hline \multirow{10}{*}{$\begin{array}{l}\text { Tâches de formulation de ques- } \\
\text { tions à partir de textes (2) }\end{array}$} & Questionnaire (1) sur une œuvre & \multirow{10}{*}{$\begin{array}{c}\text { Rédaction (2) d'un commentaire } \\
\text { Lecture d'un corpus de textes } \\
\text { courants }\end{array}$} \\
\hline & complète & \\
\hline & Modelage de la justification & \\
\hline & Questionnaires (2) & \\
\hline & Discussion entre élèves & \\
\hline & Questionnaires (2) & \\
\hline & $\begin{array}{l}\text { Lecture interactive de textes } \\
\text { courts }\end{array}$ & \\
\hline & Rétroaction interactive & \\
\hline & «Profil du lecteur $»(1)$ & \\
\hline & Entrevue avec l'enseignant[e] & \\
\hline 8 activités & 9 activités & 4 activités \\
\hline
\end{tabular}

Selon les pratiques déclarées par les enseignant[e]s, la justification est essentiellement utilisée dans trois différents contextes : pour répondre à des questions sur une ou toutes les dimensions en lecture sans enseignement de la justification ; pour répondre à des questions et être évalués en lecture avec un enseignement ou un accompagnement préalable sur la justification ; pour écrire des textes longs à la suite d'une tâche de lecture de textes.

\section{La justification pour répondre à des questions en lecture sans enseignement}

de la justification. Le premier usage dégagé du discours des enseignant[e]s et des activités présentées montre que la justification est utilisée pour travailler et pour évaluer 
une ou plusieurs dimensions de la lecture. Elle sert alors à répondre à des questions d'interprétation, de réaction ou encore d'appréciation. En effet, parmi les 21 activités analysées, 6 consistaient à lire un ou des textes courts, ou à lire un roman, et à répondre à une ou des questions portant sur une ou plusieurs dimensions de la lecture. Dans ces activités, au moins une question est utilisée pour évaluer les élèves. Ces activités n'impliquent aucun enseignement relatif à la justification et rien n'indique dans le discours des enseignant[e]s concerné[e]s qu'il y ait eu un tel enseignement ou une présentation de ce que constitue une justification. Elle semble alors correspondre à un savoir protodisciplinaire (Chevallard et Johsua, 1985/1991), c'est-à-dire un savoir considéré comme acquis par les élèves ou dont la maitrise est considérée comme allant de soi. Le terme est en fait utilisé dans la consigne fournie aux élèves : « Justifiez votre réponse ». Par ailleurs, certaines de ces activités ont impliqué, aux dires des enseignant[e]s, une étape préparatoire qui consistait, pour les élèves, à confronter, dans le cadre d'échanges oraux (par exemple, des cercles de lecture, des discussions), leur interprétation ou leur appréciation du texte, ce qui leur a permis de faire usage, du point de vue des enseignant $[\mathrm{e}] \mathrm{s}$ concerné $[\mathrm{e}] \mathrm{s}$, de la justification pour développer leur réponse.

Deux autres activités présentent une variante par rapport à celles qui viennent d'être présentées : plutôt que d'inviter les élèves à formuler des réponses justifiées, les élèves sont amenés à identifier des éléments du texte propices à l'interprétation, à la réaction ou à l'appréciation d'un aspect de l'œuvre et à formuler une question à ce propos. Les élèves doivent ensuite fournir une ou des réponses potentielles qu'ils doivent justifier. De telles tâches renversent le point de vue des élèves, alors amenés à interroger eux-mêmes le texte. À notre avis, elles permettent aux élèves de travailler plus directement les processus inhérents à l'interprétation, à la réaction et à l'appréciation en tant que telles.

Ce premier usage de la justification (répondre à des questions) s'approche de la première définition ; la justification est ici une action posée par l'énonciateur, avec parfois une prise en compte du destinataire, surtout lors des échanges. La justification sert donc à soutenir la verbalisation du raisonnement de l'élève : ce qu'il pense d'un personnage, d'un élément du texte qu'il a apprécié, etc.

\section{La justification pour répondre à des questions en lecture avec enseignement}

de la justification. Le deuxième usage déclaré de la justification et dégagé à partir des 
propos des enseignant[e]s met en lumière que cette conduite discursive est également utilisée pour répondre à des questions en lecture, mais qu'un enseignement systématique de la justification a lieu sous une forme ou une autre, faisant de la justification un savoir disciplinaire (Chevallard et Johsua, 1985/1991), c'est-à-dire un savoir à enseigner ou qui demande une attention en classe de français. Dans les activités qui présentent cet usage, la structure textuelle est formellement enseignée ou modélisée, ou alors les élèves sont guidés par l'enseignant[e] dans l'action de justifier. Que les tâches données aux élèves portent sur des textes courts ou longs, courants ou littéraires, sur une ou plusieurs dimensions en lecture, dans tous les cas, les justifications formulées à l'écrit par les élèves servent à évaluer leur compétence en lecture. Quant à celles fournies à l'oral, elles permettent aux élèves, selon les enseignant[e]s, d'apprendre à justifier et d'améliorer tant leur compétence à justifier que leur compétence à lire.

Trois activités de trois enseignant[e]s différent[e]s ont été décrites comme comportant des étapes d'enseignement systématique et formel de la structure d'une « réponse à une question ». Ces enseignant[e]s ont souligné les difficultés de leurs élèves à formuler convenablement leurs réponses écrites à des questions d'interprétation et de réaction. Leur objectif était alors de travailler la formulation des réponses écrites en présentant aux élèves les éléments essentiels d'une justification. Voici les éléments d'une « bonne justification » que présente l'une des enseignantes : 1) Réponse claire («Selon moi,... »), 2) Preuve pertinente tirée du texte, 3) Explication, 4) Reformulation/ conclusion en d'autres mots (Donc,...). Pour enseigner la justification, ces enseignant[e] $\mathrm{s}$ ont tou[te]s procédé par contraste, utilisant des exemples de réponses bien formulées et étayées à côté d'autres présentant des problèmes. Cette mise en contraste permet aux élèves, selon ces enseignant[e]s, d'observer les qualités d'une bonne justification, mais aussi de mettre des mots sur les améliorations à apporter à d'autres exemples moins réussis. Cette stratégie didactique semble d'ailleurs efficace du point de vue de ces enseignant[e]s qui constatent une amélioration de la qualité des réponses de leurs élèves à des questions d'interprétation ou de réaction. L'enseignement de la structure textuelle et de caractéristiques linguistiques de la justification contribue, selon l'avis des enseignant[e]s qui ont déclaré une telle pratique, à ce que leurs élèves parviennent à formuler des réponses plus étoffées et mieux fondées. On constate que cet usage de la justification peut être mis en parallèle avec la deuxième définition de la justification, soit celle qui la présente selon ses attributs discursifs, textuels et linguistiques. Également, 
ce travail fait auprès des élèves sur ce qu'est une bonne justification vise à les soutenir et à comprendre le critère d'évaluation « pertinence de la justification », critère utilisé, rappelons-le, pour évaluer l'interprétation, la réaction et l'appréciation en lecture (MEQ, 2006).

Parmi les activités qu'ont proposées les participant[e]s, 7 des 21 présentent des tâches portant sur plus d'une dimension de la lecture à partir de textes courts ou d'œuvres complètes, avec non pas un enseignement systématique de la structure de la justification, mais plutôt avec une forme d'accompagnement des élèves visant à mieux étayer les justifications. Six des sept activités prennent la forme de questionnaires de lecture. Deux d'entre elles sont associées à la lecture d'œuvres complètes et proposent aux élèves la réalisation individuelle, à l'extérieur du temps de classe, de courtes tâches au fil de la lecture. L'enseignante concernée dit offrir, lors de la présentation de ces activités, ce qu'elle nomme un « modelage » de la manière de s'y prendre pour justifier les réponses aux diverses questions ou aux tâches proposées. Deux autres activités consistent à accompagner les élèves dans la lecture d'un texte court auquel est joint un questionnaire. L'enseignante concernée explique procéder à une lecture interactive (avec arrêts pour clarifications, questions, échanges), puis demander aux élèves de répondre aux questions avant d'effectuer une correction en grand groupe. Ce faisant, les élèves participent aux discussions entourant l'à-propos d'une réponse, la complétude d'une autre ou la justesse d'une dernière.

Une dernière activité, qui se déploie sur plusieurs mois, vise à ce que l'élève établisse ce que l'enseignant appelle son « profil de lecteur». Pour ce faire, l'enseignant demande aux élèves de lire le début d'une œuvre, après quoi l'élève est appelé à justifier, en le consignant par écrit, son choix de poursuivre la lecture ou non. Lorsque l'élève choisit de poursuivre la lecture d'une œuvre, il doit alors entreprendre une série de tâches au fil de la lecture qui l'amènent à construire le sens de l'œuvre, puis, à terme, à en rédiger une brève appréciation. Durant toute la durée du projet, l'enseignant mène périodiquement des entretiens auprès des élèves. C'est à ces occasions que les élèves sont appelés à partager les raisons de leurs choix (donc, à justifier leurs choix) auprès de l'enseignant et à discuter de leurs lectures. Lors de ces échanges, l'enseignant demande alors des précisions, des clarifications visant entre autres à améliorer les justifications des élèves. 
Les enseignant[e]s qui ont proposé des activités impliquant une ou des formes d'interactions (entre pairs, avec l'enseignant[e] ou en grand groupe) expliquent que c'est précisément pour permettre aux élèves de faire de nouveaux apprentissages et de mieux comprendre les textes qu'ils amènent les élèves à présenter oralement leurs réponses et à les justifier. Ils précisent que les interactions entre pairs permettent à leurs élèves d'approfondir leur raisonnement, d'établir de meilleurs liens et d'interpréter, de réagir ou d'apprécier les textes de façon plus approfondie. Ces interactions provoquent en effet des réactions de la part des interlocuteurs forçant les élèves à développer leur réponse, à la reconsidérer, à mieux l'appuyer et ainsi, à développer leurs capacités de justification selon les dires des enseignant $[\mathrm{e}] \mathrm{s}$. Ces interactions permettent également, toujours selon ces enseignant $[\mathrm{e}] \mathrm{s}$, d'offrir une rétroaction à leurs élèves. Ces rétroactions portent notamment sur l'approfondissement des réponses des élèves : « pourquoi tu dis ça, quels liens as-tu faits, quel exemple du texte te permet de le dire, etc. ». Ces activités qui prévoient un enseignement de la justification couplé à des interactions visant à approfondir les réponses des élèves jumèlent en quelque sorte les deux définitions ; la justification est à la fois une action posée par l'énonciateur qui doit prendre en compte le destinataire pour faire valoir le bienfondé d'une réponse, tout en présentant les attributs textuels d'une bonne justification que l'élève est appelé à respecter dans la formulation écrite de ses réponses.

La justification dans le travail sur l'écriture de textes longs. Des activités d'écriture de textes à dominante justificative découlant d'un travail en lecture font partie des activités proposées par les enseignant[e]s. L'enseignement de la justification concerne alors plus spécifiquement l'organisation globale de ces textes (introduction, développement et conclusion typiques d'un texte à dominante justificative). Ces activités ont toutes une visée évaluative en écriture.

De notre échantillon, quatre enseignant[e]s ont proposé des activités portant sur l'écriture d'une appréciation littéraire à la suite de la lecture d'un roman, dont trois évoquent une stratégie didactique inspirée de l'approche par genres (Schneuwly et Dolz, 1997) qui consiste à étudier, à l'aide de textes exemplaires du genre, les caractéristiques génériques de l'appréciation littéraire en concomitance avec la lecture d'une œuvre. Deux autres enseignantes ont présenté des activités d'écriture préparatoires 
à l'épreuve ministérielle de la fin de la 2 e année du secondaire ${ }^{3}$ qui consiste à rédiger un texte courant de type « commentaire ». La première activité consistait en une série d'exercices de rédaction (rédaction de diverses formes d'introductions, de paragraphes de développement et de conclusions) et vise, selon l'enseignante, à aider les élèves à s'approprier la structure globale du texte. L'enseignante dit leur présenter divers modèles d'organisation afin que les élèves puissent être libres d'adopter la structure qui leur convient. Ces activités peuvent être mises en relation avec la seconde définition de la justification qui met davantage l'accent sur la structure.

La seconde activité préparatoire à l'épreuve est proposée par une enseignante dont l'objectif est de faire prendre conscience aux élèves de l'importance de bien cerner son intention d'écriture (ici, faire connaitre un personnage historique et montrer son importance et l'intérêt de le connaitre). Partant de cette intention, elle déclare amener ses élèves à lire des textes sur le personnage en question et à y relever des éléments d'information qui leur permettront de justifier l'intérêt du personnage historique. Cette manière d'utiliser l'intention de la conduite de justification (faire valoir son point) favorise à notre avis la mise en pratique d'une lecture « intentionnée » et l'établissement de liens plus concrets entre la lecture et l'écriture. Enfin, un tel travail amène les élèves à développer des processus de lecture et de planification qui sont propres au discours justificatif. De telles activités nous apparaissent prometteuses. Cette activité nous semble plus proche d'une définition qui présente la justification comme une action langagière.

Dans cette dernière section, nous relevons que les liens entre la lecture et l'écriture de textes à dominante justificative ne sont que très peu explicités dans les discours des enseignant[e]s et assez peu exploités dans les activités qu'ils [elles] proposent, sauf pour la dernière activité décrite. Pourtant, nous sommes d'avis que la qualité des textes que produisent les élèves est en bonne partie tributaire de leur compétence en lecture. En effet, les textes à dominante justificative prennent appui sur des informations tirées des textes littéraires et courants qui constituent le matériau de leurs productions écrites. Les liens entre la lecture et l'écriture restent pourtant opaques dans le discours des participant[e]s.

3 À titre d'exemple, l'épreuve de 2017 consistait à écrire un texte montrant l'intérêt, encore aujourd'hui, de tenir des expositions universelles. 


\section{Constats généraux et perspectives de recherche}

Quinze ans après l'introduction de la justification comme objet d'enseignement dans le programme québécois de français du 1er cycle du secondaire (MEQ, 2006), force est de constater qu'un flou persiste au regard de la définition que les enseignant[e]s donnent à la justification (pôle « objet»). Les deux définitions, fortement liées à celles que proposent le programme de français (MEQ, 2006) et la PDA (MELS, 2011), se côtoient et varient en fonction des usages dans le cadre du travail sur la lecture en classe de français : la justification est présentée telle une action intentionnée dans le cadre des échanges, alors qu'elle est présentée comme une structure dans le cadre des activités d'écriture servant à l'évaluation. Par ailleurs, plusieurs de ces enseignant[e]s présentent la justification sans la distinguer clairement de l'explication ou de l'argumentation, notions enseignées au 2e cycle du secondaire. Certes, des travaux doivent être menés sur la transposition didactique (Chevallard et Johsua, 1985/1991) externe et interne de la justification afin de clarifier cette notion.

Du côté des élèves (pôle « apprenants »), lorsqu'on leur présente la justification comme un outil structurant ou qu'on la provoque dans le cadre d'échanges entre pairs et avec l'enseignant, celle-ci semble avoir des retombées positives selon les propos recueillis auprès des enseignants : elle les amène à développer leur raisonnement, à faire une lecture intentionnée des textes, à mieux se connaitre comme lecteur et à apprendre à interroger les textes. C'est la première définition évoquée par les enseignant[e]s qui est alors sollicitée dans le travail en lecture qui engage la prise en charge énonciative de manière plus concrète. Selon toute vraisemblance, la conduite de justifications semble ici soutenir le développement de capacités réflexives. De telles capacités apparaissent essentielles au développement des compétences en lecture (Chabanne et Bucheton, 2002 ; Falardeau et al., 2014 ; Jaubert et Rebière, 2004 ; Sauvaire, 2015). Mais qu'en est-il de l'enseignement systématique des attributs textuels de la justification ? Cet enseignement ne sert-il qu'à formater les réponses des élèves alors plus aisées à évaluer ou soutient-il véritablement le développement de capacités réflexives, dans quelle mesure et à quelles conditions ? Encore ici, des travaux sont requis pour mieux comprendre comment l'usage de la justification à l'oral et à l'écrit pourrait devenir un levier pour le développement des compétences en lecture. 
En ce qui concerne les usages (pôle « enseignant»), les entretiens font émerger des usages somme toute assez peu variés de la justification ; la majorité des activités présentées étant catégorisables comme « questionnaires ». Bien entendu, plusieurs pratiques déclarées montrent le souci des enseignant[e]s de soutenir leurs élèves dans leurs apprentissages de la justification, de la lecture et de l'écriture : certains enseignent systématiquement la structure de la justification, d'autres s'en servent pour aider leurs élèves à approfondir leurs raisonnements, et ce, de diverses façons. Mais ils restent unanimes sur le fait que, de leur point de vue, ils ne disposent pas de suffisamment d'outils et de connaissances pour user efficacement de la justification en contexte d'enseignement de la lecture. Ils souhaiteraient varier les tâches qu'ils offrent à leurs élèves ou travailler les dimensions de la lecture de façon plus ciblée.

Les analyses ont enfin montré que les liens entre la lecture de textes et l'écriture de commentaires (textes à dominante justificative) sont pratiquement absents du discours des enseignant[e]s. Ainsi, il nous semble essentiel de mener des recherches en didactique du français selon trois objectifs : 1) concevoir et mettre à l'essai des dispositifs didactiques qui lient la lecture et l'écriture de textes à dominante justificative (Forget, $2014)$;2) comprendre quels seraient les gestes didactiques propres à l'enseignement conjoint de la justification et de la lecture les plus porteurs ; 3) poursuivre les travaux sur des pratiques d'enseignement de la lecture en appui sur les interactions orales qui intègrent la justification (Hébert et al., 2015 ; Hébert et Lafontaine, 2012 ; Sénéchal, 2016). Partant de cette recherche exploratoire, nous nous proposons de poursuivre en ce sens, avec l'hypothèse selon laquelle la conduite justificative s'avère un outil didactique rempli de promesses. 


\section{Références}

Ahr, S. et Joole, P. (dir.). (2013). Carnet/journal de lecteur/lecture : Quels usages, pour quels enjeux, de l'école à l'université ? Presses universitaires de Namur.

Chabanne, J.-C. et Bucheton, D. (dir.). (2002). Parler et écrire pour penser, apprendre et se construire : L'écrit et l'oral réflexifs. Presses universitaires de France. https:// doi.org/10.3917/puf.chaba.2002.01

Chartrand, S.-G. (2013). Enseigner à justifier ses propos de l'école à l'université. Correspondance, 19(1). http://correspo.ccdmd.qc.ca/Corr19-1/2.html

Chevallard, Y. et Johsua, M.-A. (1985/1991). La transposition didactique. Du savoir savant au savoir enseigné. La Pensée sauvage.

Dabène, M. (1991). Un modèle didactique de la compétence scripturale. Repères, (4), 9-22. https://www.persee.fr/doc/reper_1157-1330_1991_num_4_1_2030

Dabène, M. (2005). Quelques repères, perspectives et propositions pour une didactique du français dans tous ses états. Dans J.-L. Chiss, J. David et Y. Reuter (dir.), Didactique du français : Fondements d'une discipline (p. 15-34). De Boeck.

Falardeau, É. (2003). Compréhension et interprétation : deux composantes complémentaires de la lecture littéraire. Revue des sciences de l'éducation, 29 (3), 673-694. https://doi.org/10.7202/011409ar

Falardeau, É., Simard, D., Sauvaire, M. et Gagné, J.-C. (2014). L’activité réflexive d'élèves du secondaire analysée sous l'angle de ses manifestations langagières à l'oral. Revue suisse des sciences de l'éducation, 36 (2), 333-350.

Fasel-Lauzon, V. (2009). L'explication dans les interactions en classe de langue : organisation des séquences, mobilisation de ressources, opportunités d'apprentissage [Thèse de doctorat, Université de Neuchâtel]. https://doc.rero.ch/record/12606/files/Th_FaselV.pdf

Fisher, C. et Nadeau, M. (2014). Usage du métalangage et des manipulations syntaxiques au cours de dictées innovantes dans des classes du primaire. Repères, (49), 169192. https://doi.org/10.4000/reperes.742

Forget, M.-H. (2012). Apprendre à justifier ses propos au secondaire : quels savoirs enseigner, quelle progression proposer? Dans J.-L. Dumortier, J. Van Beveren et 
D. Vrydaghs (dir.), Curriculum et progression en français. Actes du 11e colloque de l'AIRDF (p. 177-191). Presses universitaires de Namur.

Forget, M.-H. (2014). Pratiques d'écriture de justifications d'élèves plurilingues du ler cycle du secondaire. Entre savoirs d'expérience et apprentissages en classe de français [Thèse de doctorat, Université de Sherbrooke]. http://hdl.handle.net/11143/5373

Forget, M.-H. (2017). Tactiques d'élaboration de justifications écrites d'élèves du secondaire. Recherches en didactiques, (24), 41-60.

Garcia-Debanc, C. (1994). Apprendre à justifier par écrit une réponse : analyses linguistiques et perspectives didactiques. Pratiques, (84), 5-40. https://www. persee.fr/doc/prati_0338-2389_1994_num_84_1_1725

Garcia-Debanc, C. (1996). Apprendre à justifier à l'école et au collège : ruptures ou continuité ? Dans J. David et S. Plane (dir.), L'apprentissage de l'écriture de l'école au collège (p. 105-130). Presses universitaires de France.

Hébert, M., Guay, A. et Lafontaine, L. (2015). Reformuler et justifier les propos dans les cercles de lecture après enseignement de l'oral : différences entre élèves et perception des enseignants. Language and Literacy, 17(4), 28-51. https://doi. org/10.20360/G2GC78

Hébert, M. et Lafontaine, L. (2012). L'oral réflexif dans les cercles de lectures littéraires entre pairs : analyse des conduites de justifications en interaction. Dans R. Bergeron et G. Plessis-Bélair (dir.), Représentations, analyses et descriptions du français oral, de son utilisation et de son enseignement au primaire, au secondaire et à l'université (p. 201-219). Éditions Peisaj.

Husserl, E. (1992). Méditations cartésiennes. Vrin.

Jaubert, M. et Rebière, M. (2004, 26-28 aout). Quelques fonctions de l'activité langagières en français [Communication]. 9e colloque de 1'AIRDF, Québec.

Jorro, A. (1999). Le lecteur interprète. Presses universitaires de France. https://doi. org/10.3917/puf.jorro.1999.01

Karsenti, T. et Savoie-Zajc, L. (2004). La recherche en éducation : étapes et approches. Éditions du CRP. 
Krief, N. et Zardet, V. (2013). Analyse de données qualitatives et recherche-intervention. Recherches en sciences de gestion, 2(95), 211-237. https://doi.org/10.3917/ resg.095.0211

Ministère de l'Éducation du Québec [MEQ]. (2001). Programme de formation de l'école québécoise. Éducation préscolaire et enseignement primaire. http://www. education.gouv.qc.ca/fileadmin/site web/documents/education/jeunes/pfeq/ PFEQ presentation-primaire.pdf

Ministère de l'Éducation du Québec [MEQ]. (2006). Programme de formation de l'école québécoise : Enseignement secondaire, premier cycle. https://www.aqpde.ca/ wp-content/uploads/2011/07/Programme-de-formation-secondaire-1er-cycle.pdf

Ministère de l'Éducation, du Loisir et du Sport [MELS]. (2011). La progression des apprentissages au secondaire : Français, langue d'enseignement. http://www. education.gouv.qc.ca/fileadmin/site_web/documents/education/jeunes/pfeq/PDA PFEQ francais-langue-enseignement-secondaire_2011.pdf

Paillé, P. et Mucchielli, A. (2016). L'analyse qualitative en sciences humaines et sociales. Armand Colin.

Sauvaire, M. (2015). La diversité des sujets lecteurs dans l'enseignement de la lecture littéraire. Éducation et didactique, 9(2), 107-117. https://doi.org/10.4000/ educationdidactique. 2310

Schneuwly, B. et Dolz, J. (1997). Les genres scolaires. Des pratiques langagières aux objets d'enseignement. Repères, (15), 27-40. https://doi.org/10.3406/ reper.1997.2209

Sénéchal, K. (2016). Expérimentation et validation de séquences didactiques produites selon une ingénierie didactique collaborative. L'enseignement de la discussion et de l'exposé critique au secondaire [Thèse de doctorat, Université Laval]. http:// hdl.handle.net/20.500.11794/27225

Tallet, C. (2003). « Faut pas imaginer, faut voir la réalité » - Rémi (9 ans) ou Comment les activités métalinguistiques peuvent-elles aider les élèves à passer d'une écriture inventée à une analyse formelle de la langue ? Le cas de l'accord sujetverbe chez les enfants de CE2. Repères, (28), 27-46. https://www.persee.fr/doc/ reper 1157-1330_2003 num 28_1_2420 